\title{
INTERCAMBIANDO EXPERIENCIAS EN GRUPOS ONLINE: ANÁLISIS DEL ESTADO EMOCIONAL DE LOS PADRES DE HIJOS CON DISCAPACIDAD
}

\section{Exchanging experiences on online groups: analysis of the emotional state of the parents of children with disabilities}

\author{
Raquel Suriá MarTíneZ \\ Universidad de Alicante. Departamento de Comunicación y Psicología Social. Edificio de Cien- \\ cias Sociales. Carretera San Vicente del Raspeig, s/n. 03690 San Vicente del Raspeig. Alicante \\ raquel.suria@ua.es
}

Recepción: 7 de marzo de 2017

Aceptación definitiva: 12 de septiembre de 2017

Resumen: Conocer el estado anímico de los progenitores de hijos con discapacidad puede ser importante para su bienestar. Este estudio se dirige a comparar el contenido emocional intercambiado de 460 mensajes enviados por 63 participantes a los foros online dedicados a padres y madres de hijos con discapacidad en función del momento en el que se adquiere la discapacidad, de ellos, 230 procedentes de padres de hijos con discapacidad adquirida desde el nacimiento y 230 de progenitores de hijos con discapacidad sobrevenida, así como en función del género de estos progenitores. Los resultados de los análisis multivariados de varianza mostraron que los progenitores varones de hijos con discapacidad sobrevenida indicaron tener un estado de ánimo y emocional más negativo que los participantes de hijos con discapacidad adquirida. Esto sugiere que la adquisición de la discapacidad es una variable que puede repercutir en la satisfacción de vida de los padres y madres de hijos con discapacidad, por tanto, es importante tenerla en cuenta en los programas de intervención.

Palabras Clave: grupos virtuales; padres de hijos con discapacidad; análisis de contenido; estado emocional. 
AвSTRACT: Knowledge about the physical state of the parents of children with disabilities is important for their well-being. The objetive of this study aims to compare the emotional content exchanged in 460 messages sent to the online forums related to parents of children with disabilities according to the moment where the disability is acquired, 230 from parents of children with disabilities acquired from birth and 230 from parents of children with sudden acquired disability, and gender of these parents. The results of the multivariate analyzes of variance showed that the male parents of children with an impaired disability indicated a more negative emotional and mood state than the participants with acquired from birth disability. This suggests that the moment in wich the disability is acquired is a factor that can affect the life satisfaction of parents of children with disabilities, therefore it is important to take into account in intervention programs.

KEY WORDS: virtual groups; parents of children with disabilities; LIWC; emotional state.

\section{Introducción}

$\longrightarrow$ ESDE HACE ALGUNOS AÑOS, el interés por diseñar programas de intervención hacia las familias de personas con discapacidad se ha incrementado de forma notable (Benítez y Soto, 2012; Dunst y Trivette, 2009; Jordán de Urríes, 1997; Schalock, Bohman y Verdugo, 2008; Suriá, 2013).

Esto principalmente hace que la familia, y concretamente los progenitores de los hijos con discapacidad, sean el objetivo prioritario de intervención, ya que, en la mayoría de ocasiones, estos progenitores van a tener que enfrentarse a nuevas situaciones y a cambios en sus roles, que pasan desde ser los cuidadores, celadores, enfermeros e incluso, a veces, médicos, sin recibir el apoyo y ayuda adecuados para enfrentarse a esta situación (Benítez y Soto, 2012; Samios, Pakenham y Sofronoff, 2009; Smith, Hong, Seltzer, Greenberg, Almeida y Bishop, 2010).

Así, aunque todo esto lo lleven a cabo pensando en el bienestar de los hijos con discapacidad, en muchas situaciones, puede derivar en cambios en el estado anímico y emocional, sentimientos de negación, ansiedad y otros problemas que van a repercutir en la salud y en la adaptación y convivencia de los afectados, así como de todos los miembros de la familia. Por ejemplo, algunas investigaciones muestran los efectos traumáticos de la crianza de hijos con discapacidad en los progenitores (Dabrowska y Pisula, 2010; Jordán de Urríes, 1997; Lequerica, Forchheimer, Albright,Tate, Duggan y Rahman, 2010; Singer, Ethridge y Sandra, 2007), principalmente en madres (Olsson y Hwang, 2008), en las que la depresión (Azar y Kurdahi, 2006), estrés o sobrecarga (Suriá, 2011) son el común denominador.

Sin embargo, ¿cómo es que algunas familias muestran una mayor adaptación y bienestar en la experiencia de tener un hijo con discapacidad?, ¿qué variables son las que influyen en una adaptación exitosa?, ¿ se ajustan más positivamente los padres o las madres que cuidan a los hijos con esta problemática?

Para dar respuesta a esto, un amplio volumen de investigaciones sobre adaptación familiar y discapacidad se ha fundamentado en variables inherentes a la discapacidad, 
como pueden ser la tipología (Dabrowska y Pisula, 2010) o el grado de severidad de esta (Krethong, Jirapaet, Jitpanya y Sloan, 2008), que son factores que cobran un especial protagonismo. Así, por ejemplo, se ha demostrado que existen perfiles diferenciales en el grado de ajuste de la familia en función de la tipología de la discapacidad. Autores como Dabrowska y Pisula (2010) examinaron a padres con hijos de dos tipos diferentes de discapacidades del desarrollo, hijos con autismo e hijos con síndrome de Down. Los resultados indicaron mayor nivel de bienestar parental entre los padres de niños con síndrome de Down que los de hijos con autismo.

También con fines comparativos, Amaya, Celene, Acle Tomasini, Ampudia Rueda y García Méndez (2014) encontraron sentimientos de negación y estados de ánimo negativos en las familias de hijos cuya discapacidad era de etiología desconocida con respecto a las familias de hijos con síndrome de Down.

Si bien en los estudios citados anteriormente se examinan los cambios en el estado emocional y en el ajuste y adaptación parental de hijos con discapacidad, todos se basan en las diferencias en funcion de la tipologia de la discapacidad, no teniendose en cuenta una variable muy influyente: el momento en el que los hijos adquirieren este hándicap (Uppal, 2006; Ford, Gerryoe y Tennant, 2001; Suriá, 2013).

A su vez, al atender a los estudios dedicados a conocer el impacto y estado emocional de los padres y madres que cuidan de hijos con discapacidad, la mayoría de estudios han acudido a cuestionarios o autoinformes (Dabrowska y Pisula, 2010; Jordán de Urríes, 1997; Suriá, 2013; Urzúa, Godoy y Ocayo, 2011), bien averiguándolo de forma directa, es decir, preguntándole a los padres o bien a través de los familiares y profesionales implicados en el día a día de estas personas.

Sin embargo, otra forma de conocer el impacto emocional de los progenitores de hijos con discapacidad es por medio de la escritura. Así, existe cada vez más evidencia de que las palabras que se utilizan para expresarnos permiten identificar indicadores significativos del estado de ánimo y emocional (Pennebaker Francis y Booth, 2001; Pennebaker, Mehl y Niederhoffer, 2003). Por ejemplo, Rude, Gortner y Pennebaker (2004) analizaron textos que habían escrito estudiantes deprimidos y estudiantes no deprimidos. Se encontró que los estudiantes deprimidos utilizaban más palabras con connotaciones negativas y menos palabras referentes a aspectos positivos que los estudiantes no deprimidos.

En otro estudio, Stirman y Pennebaker (2001) compararon el uso del idioma (pronombres, verbos, etc.) en poetas que cometieron suicidio y no suicidas. Se encontró que los poetas que se habían suicidado utilizaban menos palabras en primera persona del plural (p. e., nosotros) y más palabras en primera persona del singular (p. e., yo). A su vez, los poetas suicidas tendieron a utilizar menos palabras referentes a temas relacionados con los amigos y la familia y más palabras asociadas con la muerte.

A partir de esta idea se sugiere que las palabras que utiliza una persona durante su discurso podrían permitir una mayor comprensión del significado subjetivo que tiene dicha persona sobre sí misma y su satisfacción con el entorno ( Schwartz et al., 2013; Slatcher, Vazire y Pennebaker, 2008; Stirman y Pennebaker, 2001).

Actualmente, el máximo representante del estudio del uso del lenguaje como “marcador psicológico” es el grupo de James Pennebaker. Este autor parte de la idea 
de que a través de los estilos lingüísticos se refleja el estado de ánimo de las personas, así como sus pensamientos.

Basándose en esto, diseñó el programa informático LIWC (Linguistic Inquiry and Word Count), una herramienta de análisis de textos que se basa en el recuento de palabras y las categoriza en función de diversas dimensiones: procesos psicológicos, preocupaciones o asuntos personales, dimensiones lingüísticas, etc. (Pennebaker et al., 2001).

En relación a esto, y exceptuando a Carrol (2008, 2009), que examinó el patrón emocional de los padres y madres de hijos con discapacidad intelectual, y en el que se observaron mayores emocioes negativas en los progenitores varones, la mayoría de investigaciones se han centrado en el estudio diferencial del uso del lenguaje escrito, en personas con diferentes enfermedades físicas (Alpers et al., 2005; Suriá y Beléndez, 2009; van Uden-Kraan et al., 2008) y mentales (Beléndez y Suriá, 2011; RamírezEsparza, Chung, Sierra-Otero y Pennebaker, 2009).

Si bien es cierto que esto se ha llevado a cabo a través de narraciones tanto orales (Carrera-Fernández, Olmos y Peró, 2013; Cavicchio y Poesio, 2012; Valdés-Sánchez, 2010), como escritas (Carroll, 2008; Graesser, Dowell y Moldovan, 2011; Robinson, Navea y Ickes, 2013), en los últimos años, internet ha posibilitado otra forma de conocer las expresiones emocionales y el estado de ánimo que se intercambia por escrito.

Este es el caso de las comunidades o grupos virtuales, entornos online, utilizados por multitud de padres y madres de hijos con discapacidad para intercambiar sus experiencias y emociones a través de sus contribuciones (Stephen y Keating, 2011; Wright, Rains y Banas, 2010). Estos espacios, además de propiciar entre sus participantes una expresión emocional más abierta, así como mayores posibilidades de desvelar información personal anonimamente a través de los mensajes, ofrecen otra forma de obtener información sobre el estado de ánimo y emocional que reflejan los usuarios participantes en las narraciones que escriben.

Con el propósito de conocer de forma más exhaustiva lo que significa la experiencia de cuidar a un hijo con discapacidad, en este estudio se analiza el estilo emocional de los mensajes enviados a diversos foros de internet destinados a los progenitores que cuidan a un hijo con discapacidad; esto se analiza en función de la adquisición de la discapacidad de los hijos y del género de los progenitores.

Así, los objetivos especificos se concretan en los siguientes:

1. Examinar si existen diferencias estadísticamente significativas en las expresiones escritas, esto es, en el lenguaje intercambiado en los mensajes enviados a los foros en función del tipo de foro (foro de discapacidad desde el nacimiento vs. foro de discapacidad sobrevenida). De este objetivo se desprende que:

H1. Los mensajes intercambiados en foros dirigidos a padres de hijos con discapacidad sobrevenida a lo largo de la vida tendrán connotaciones más negativas que los mensajes enviados a foros de padres de hijos con discapacidad adquirida en el nacimiento.

2. Conocer si existen diferencias estadísticamente significativas en las expresiones escritas intercambiadas en los foros en función del género de los autores de los mensajes (masculino vs. femenino). De este objetivo se espera que: 
H2. Las contribuciones intercambiadas en los foros escritas por varones tendrán connotaciones más positivas que las contribuciones intercambiadas por mujeres.

\section{Método}

\subsection{Participantes}

La muestra estuvo compuesta por los mensajes enviados por los participantes a 6 foros. En total, se han recopilado 460 mensajes a varios foros dirigidos a padres y madres de hijos con discapacidad, 230 mensajes enviados a 3 foros dirigidos a padres de hijos con discapacidad adquirida desde el nacimiento (espina bífida y parálisis cerebral) y 230 a 3 foros destinados a padres de hijos con discapacidad sobrevenida a lo largo de la vida (lesión medular y esclerosis múltiple). Con respecto al género de los participantes que intercambiaron mensajes en los foros dirigidos a padres de hijos con discapacidad adquirida desde el nacimiento, 310 de las contribuciones fueron escritas por 34 mujeres y 150 fueron escritas por 29 hombres.

\subsection{Procedimiento}

Inicialmente se seleccionaron foros en los que la actividad de participación de los usuarios fuera frecuente. Se recopilaron mensajes durante 4 meses y fueron revisados ortográficamente para que el programa identificara el porcentaje máximo de palabras. A su vez, fueron eliminados aquellos mensajes en los que el género de sus autores no era identificable.

Para analizar el estilo lingüístico y estado emocional de los mensajes de los participantes el instrumento utilizado ha sido el programa informatizado Linguistic Inquiry Word Count, LIWC (Pennebaker et al., 2001), en su versión castellana (RamírezEsparza, García, Pennebaker y Suriá, 2007). El programa contiene un diccionario interno de 7000 palabras enumeradas con valores arbitrarios y su funcionamiento se basa en clasificar las palabras de un texto en base a 72 dimensiones pertenecientes a diversas dimensiones: procesos psicológicos, preocupaciones o asuntos personales, dimensiones lingüísticas (pronombres, etc.) y, a continuación, calcular el porcentaje de palabras que pertenecen a cada categoría y subcategoría. Las categorías del LIWC de interés seleccionadas fueron 1) los aspectos emocionales (positivos y negativos), 2) tiempos verbales y 3 ) pronombres.

\subsection{Análisis estadístico}

Para comprobar si existían diferencias a nivel estadísticamente significativo se utilizaron análisis multivariados de varianza (MANOVAS), teniendo dos variables 
independientes (tipo de foro y género de los progenitores) y tres variables dependientes (estado de ánimo, tiempo verbal y pronombres). Para utilizar esta prueba paramétrica previamente se comprobaron los supuestos de normalidad, independencia y homogeneidad de varianzas. De manera complementaria se calculó el tamaño del efecto a través de la $d$ de Cohen (1988) para calcular la magnitud de las diferencias observadas.

Una vez recolectados los datos se analizaron por medio del paquete estadístico SPSS versión 19.0.

\section{Resultados}

Al examinar si existían diferencias en las dimensiones analizadas, esto es, expresiones emocionales, tiempos verbales y pronombres, se observaron los siguientes resultados:

En relación a las dimensiones emocionales de los padres y madres de hijos con discapacidad, en función de la adquisición de la discapacidad en la que los hijos adquirieron esta, los resultados del MANOVA fueron estadísticamente significativos (Lambda de Wilks ${ }_{0,452} F=45,290, p<, 001$ ). Del mismo modo, el análisis multivariado para la variable género resultó estadísticamente significativo (Lambda de Wilks ${ }_{0,939} F$ $=2,422, p<, 001)$.

Con respecto a los resultados de los MANOVAS dirigidos a examinar el tiempo verbal según la adquisición de discapacidad de los hijos (Lambda de Wilks ${ }_{0,910} F=$ $5,965, p<, 001$ ) y el género de los progenitores (Lambda de Wilks ${ }_{0,982} F=1,129, p<$ ,005), también fueron significativos. Lo mismo indicaron los análisis multivariados en el uso de pronombres para estas variables, es decir, para el momento en que adquirieron la discapacidad (Lambda de $\left.\mathrm{Wilks}_{0,983} F=1,704, p<, 005\right)$ y el género (Lambda de Wilks $\left._{0,992} F=0,775, p<, 005\right)$.

$\mathrm{Al}$ analizar las dimensiones emocionales positivas de los progenitores, en las que se observaron diferencias estadísticamente significativas en función de la adquisición de la discapacidad, los índices medios más elevados se encontraron en los mensajes intercambiados en los foros de discapacidad adquirida desde el nacimiento tanto en el afecto, $t(308)=2,318, p<, 05$; como en las emociones positivas, $t(308)=8,328$, $\mathrm{p}$ $<, 05$; en los sentimientos positivos, $t(308)=8,085, p<, 05$; y en el optimismo, $t(308)$ $=24,372, p<, 001$ (Tabla 1). En cuanto a los estados emocionales negativos se observó que fueron los mensajes enviados por los progenitores participantes de los foros de discapacidad sobrevenida los que indicaron medias más elevadas en las emociones negativas, $t(308)=3,297, p<, 05$, así como en el miedo, $t(308)=2,882, p<, 05$ y el enfado, $t(308)=7,923, p<, 05$ (Tabla 1$)$.

Con respecto al estado de ánimo de los padres y madres de hijos con discapacidad en función del género, los resultados indicaron que, en algunos procesos emocionales positivos, fueron las mujeres las que mayor porcentaje de palabras de esta índole intercambiaron en sus mensajes. Esto se observó en el afecto, $t(308)=3,017, p<, 05$; en los sentimientos positivos, $t(308)=0,436, p<, 05$, y en el optimismo, $t(308)=1,363, p<$ ,05 (Tabla1). En lo referente a las dimensiones negativas, se observó que los varones 
mostraron puntuaciones medias más altas que las mujeres en la negatividad, $t(308)=$ 0,540, $p<, 05$, así como en el enfado, $t(308)=10,291, p<, 05$ (Tabla 1).

\begin{tabular}{|c|c|c|c|c|c|c|c|c|c|c|c|}
\hline \multicolumn{12}{|c|}{$\begin{array}{c}\text { TaBla 1. Dimensiones emocionales según la adquisición de la discapacidad } \\
\text { y del género de los progenitores }\end{array}$} \\
\hline \multirow{2}{*}{$\begin{array}{l}\text { Dimensiones } \\
\text { emocionales }\end{array}$} & \multirow{2}{*}{\begin{tabular}{|c|} 
Género \\
$\begin{array}{c}\text { Etapa } \\
\text { discapacidad }\end{array}$
\end{tabular}} & \multicolumn{2}{|c|}{ Mujer } & \multicolumn{2}{|c|}{ Varón } & \multicolumn{2}{|c|}{ Total } & \multirow[b]{2}{*}{$\begin{array}{c}t \\
\text { etapa }\end{array}$} & \multirow[b]{2}{*}{$d$} & \multirow{2}{*}{$\stackrel{t}{t}$} & \multirow[b]{2}{*}{$d$} \\
\hline & & M & DT & M & DT & M & DT & & & & \\
\hline \multirow{3}{*}{ Afecto } & Adquirida & 7,25 & 3,42 & 6,03 & 3,00 & 9,39 & 3,38 & \multirow{3}{*}{$2,318^{*}$} & \multirow{3}{*}{0,025} & & \\
\hline & Sobrevenida & 5,67 & 3,09 & 6,16 & 3,05 & 6,23 & 3,06 & & & $3,017 *$ & 0,20 \\
\hline & Total & 6,15 & 3,36 & 6,19 & 3,05 & 7,31 & 3,22 & & & & \\
\hline \multirow{3}{*}{$\begin{array}{l}\text { Emociones } \\
\text { positivas }\end{array}$} & Adquirida & 5,31 & 4,06 & 5,22 & 4,22 & 5,30 & 4,07 & \multirow{3}{*}{$8,328^{*}$} & \multirow{3}{*}{0,007} & 0,016 & 0,001 \\
\hline & Sobrevenida & 3,58 & 2,47 & 3,81 & 2,64 & 3,77 & 2,60 & & & & \\
\hline & Total & 5,01 & 3,89 & 4,00 & 2,92 & 4,53 & 3,50 & & & & \\
\hline \multirow{3}{*}{$\begin{array}{l}\text { Sentimientos } \\
\text { positivos }\end{array}$} & Adquirida & 3,44 & 4,29 & 2,69 & 3,88 & 3,35 & 4,24 & \multirow{3}{*}{$8,085^{*}$} & \multirow{3}{*}{0,002} & $0,436 *$ & 0,021 \\
\hline & \begin{tabular}{|l|} 
Sobrevenida \\
\end{tabular} & 1,55 & 1,78 & 1,62 & 1,87 & 1,61 & 1,85 & & & & \\
\hline & Total & 3,12 & 4,03 & 1,77 & 2,26 & 2,48 & 3,38 & & & & \\
\hline \multirow{3}{*}{ Optimismo } & Adquirida & 10,49 & 4,59 & 8,07 & 3,96 & 9,65 & 6,53 & \multirow{3}{*}{$24,372 \%$} & \multirow{3}{*}{0,380} & $1,363 \%$ & 0,041 \\
\hline & Sobrevenida & 1,02 & 1,40 & ,96 & 1,04 & 1,97 & 2,11 & & & & \\
\hline & Total & 7,20 & 5,07 & 2,16 & 3,49 & 4,81 & 5,06 & & & & \\
\hline \multirow{3}{*}{$\begin{array}{l}\text { Emociones } \\
\text { negativas }\end{array}$} & Adquirida & 2,29 & 2,05 & 1,68 & 1,95 & 2,91 & 2,04 & \multirow{3}{*}{$3,297 *$} & \multirow{3}{*}{0,101} & $0,540 \%$ & 0,004 \\
\hline & Sobrevenida & 2,39 & 1,91 & 2,86 & 1,63 & 5,78 & 1,69 & & & & \\
\hline & Total & 2,31 & 2,02 & 3,70 & 1,72 & 2,99 & 1,89 & & & & \\
\hline \multirow{3}{*}{ Ansiedad } & Adquirida & 1,13 & 1,31 & 0,90 & 1,06 & 1,10 & 1,28 & \multirow{3}{*}{1,092} & \multirow{3}{*}{0,004} & 0,222 & 0,000 \\
\hline & Sobrevenida & 1,31 & 1,54 & 1,61 & 1,90 & 1,55 & 1,84 & & & & \\
\hline & Total & 1,16 & 1,35 & 1,51 & 1,82 & 1,33 & 1,60 & & & & \\
\hline \multirow{3}{*}{ Miedo } & Adquirida & 0,29 & 0,61 & 0,77 & 1,69 & 0,35 & 0,84 & \multirow{3}{*}{$2,882^{*}$} & & 0,001 & 0,000 \\
\hline & Sobrevenida & 2,26 & 2,89 & 1,76 & 2,38 & 1,85 & 2,47 & & 0,009 & & \\
\hline & Total & 0,63 & 1,50 & 1,62 & 2,32 & 1,10 & 1,99 & & & & \\
\hline & Adquirida & 0,26 & 0,65 & 0,14 & 0,38 & 1,25 & 0,62 & & & & \\
\hline Enfado & Sobrevenida & 3,78 & 2,56 & 3,86 & 2,82 & 4,21 & 2,86 & $7,923 *$ & 0,065 & $10,291 \%$ & 0,033 \\
\hline & Total & 1,04 & 2,09 & 2,89 & 2,78 & 1,73 & 2,54 & & & & \\
\hline
\end{tabular}

$*<, 05 ; * *<, 001$.

Al examinar el tiempo verbal, los mensajes enviados a los foros de padres de hijos con discapacidad sobrevenida en pasado fueron más frecuentes que los intercambiados en los foros dirigidos a padres de hijos con discapacidad desde el nacimiento, $t$ $(308)=2,631, p<, 05$. Asimismo, se observó que los progenitores varones escribieron más que las mujeres en pasado, $t(308)=2,130, p<, 05$ (Tabla 2). 


\begin{tabular}{|c|c|c|c|c|c|c|c|c|c|c|c|}
\hline \multirow[b]{2}{*}{ Tiempo verbal } & Género & Mujer & & \begin{tabular}{|l|} 
Varón \\
\end{tabular} & & Total & & \multirow{2}{*}{$\begin{array}{c}\mathrm{t} \\
\text { etapa }\end{array}$} & \multirow[b]{2}{*}{$\mathrm{d}$} & \multirow[b]{2}{*}{$\begin{array}{c}\mathrm{t} \\
\text { género }\end{array}$} & \multirow[b]{2}{*}{ d } \\
\hline & $\begin{array}{c}\text { Etapa } \\
\text { discapacidad }\end{array}$ & $\mathrm{M}$ & DT & $\mathrm{M}$ & DT & $\mathrm{M}$ & DT & & & & \\
\hline \multirow{3}{*}{ Pasado } & Adquirida & 1,93 & 2,56 & 4,24 & 1,92 & 1,84 & 2,49 & \multirow{3}{*}{$2,631 *$} & \multirow{3}{*}{0,038} & & \\
\hline & Sobrevenida & 2,92 & 2,02 & 1,09 & 1,91 & 2,65 & 2,13 & & & $2,130 \%$ & 0,007 \\
\hline & Total & 1,93 & 2,47 & 3,54 & 1,91 & 1,75 & 2,22 & & & & \\
\hline \multirow{3}{*}{ Presente } & Adquirida & 10,56 & 3,93 & 10,59 & 4,12 & 10,56 & 3,94 & \multirow{3}{*}{0,094} & \multirow{3}{*}{0,009} & & \\
\hline & Sobrevenida & 11,81 & 4,92 & 11,48 & 3,86 & 11,54 & 4,06 & & & 0,054 & 0,000 \\
\hline & Total & 10,77 & 4,13 & 11,36 & 3,89 & 11,05 & 4,02 & & & & \\
\hline \multirow{3}{*}{ Futuro } & Adquirida & 0,56 & 0,92 & 0,54 & 0,82 & 0,56 & 0,91 & \multirow{3}{*}{0,132} & \multirow{3}{*}{0,007} & & \\
\hline & Sobrevenida & 0,79 & 1,02 & 0,76 & 0,96 & 0,76 & 0,96 & & & 0,033 & 0,000 \\
\hline & Total & 0,60 & 0,94 & 0,73 & 0,94 & 0,66 & 0,94 & & & & \\
\hline
\end{tabular}

$*<, 05 ; * *<, 001$.

En referencia a los pronombres se observó mayor frecuencia de palabras en primera persona del singular, $t(308)=15,434, p<, 001$, y en segunda persona del singular y del plural, $t(308)=7,324, p<, 001$, en los mensajes enviados a los foros dedicados a padres de hijos con discapacidad sobrevenida a lo largo de la vida. Con respecto al uso de pronombres y el género, se observó que la mayoría de mensajes en primera persona del singular procedían del género masculino, $t(308)=3,204, p<, 05$ (Tabla 3).

\begin{tabular}{|c|c|c|c|c|c|c|c|c|c|c|c|}
\hline \multicolumn{12}{|c|}{$\begin{array}{l}\text { Tabla 3. Pronombres según la adquisición de la discapacidad y del género } \\
\text { de los progenitores }\end{array}$} \\
\hline \multirow[b]{2}{*}{ Pronombres } & \multirow{2}{*}{\begin{tabular}{|c|} 
Género \\
$\begin{array}{c}\text { Etapa } \\
\text { discapacidad }\end{array}$ \\
\end{tabular}} & \multicolumn{2}{|c|}{ Mujer } & \multicolumn{2}{|c|}{ Varón } & \multicolumn{2}{|c|}{ Total } & \multirow[b]{2}{*}{$\begin{array}{c}\mathrm{t} \\
\text { etapa }\end{array}$} & \multirow[b]{2}{*}{$\mathrm{d}$} & \multirow{2}{*}{ género } & \multirow[b]{2}{*}{ d } \\
\hline & & M & DT & M & DT & M & DT & & & & \\
\hline \multirow{3}{*}{ Yo } & Adquirida & 4,09 & 4,36 & 6,88 & 4,60 & 5,93 & 4,39 & \multirow{3}{*}{$15,434^{*}$} & \multirow{3}{*}{0,048} & & \\
\hline & Sobrevenida & 3,59 & 2,70 & 5,41 & 3,68 & 7,26 & 3,53 & & & $3,204 \%$ & 0,40 \\
\hline & Total & 6,49 & 4,32 & 4,61 & 3,83 & 5,59 & 4,20 & & & & \\
\hline \multirow{3}{*}{ Nosotros } & Adquirida & 0,33 & 0,87 & 0,60 & 1,42 & 0,37 & 0,95 & \multirow{3}{*}{1,455} & \multirow{3}{*}{0,005} & & \\
\hline & Sobrevenida & 0,53 & 0,93 & 0,82 & 1,29 & 0,77 & 1,23 & & & 0,096 & 0,08 \\
\hline & Total & 0,37 & 0,88 & 0,79 & 1,30 & 0,57 & 1,12 & & & & \\
\hline \multirow{3}{*}{ Tú, vosotros } & Adquirida & 0,94 & 1,55 & 0,62 & 2,80 & 0,98 & 6,75 & \multirow{3}{*}{$7,324 *$} & \multirow{3}{*}{0,023} & & \\
\hline & Sobrevenida & 2,08 & 2,56 & 2,70 & 1,87 & 1,77 & 2,01 & & & 0,022 & 0,01 \\
\hline & Total & 1,14 & 1,80 & 1,64 & 2,02 & 1,37 & 2,92 & & & & \\
\hline \multirow{3}{*}{ Él, ellos } & Adquirida & 7,37 & 4,22 & 6,41 & 4,26 & 7,24 & 4,22 & \multirow{3}{*}{14,147} & \multirow{3}{*}{0,044} & & \\
\hline & Sobrevenida & 4,08 & 3,04 & 5,10 & 3,50 & 4,92 & 3,44 & & & 0,027 & 0,004 \\
\hline & Total & 6,80 & 4,22 & 5,28 & 3,62 & 6,08 & 4,02 & & & & \\
\hline
\end{tabular}

$*<, 05 ; * *<, 001$.

(c) Ediciones Universidad de Salamanca / CC BY-NC-ND

Siglo Cero, vol. 49 (2), n. ${ }^{\circ}$ 266, 2018, abril-junio, pp. 59-72

$$
-66-
$$




\section{Discusión}

En este estudio se compara el contenido emocional que los padres y madres de hijos con discapacidad reflejan en los mensajes intercambiados en los foros virtuales en función del momento en el que se adquiere la discapacidad. Paralelamente se analiza si el género de los progenitores, ser padre o madre, influye en las emociones expresadas. Así, fundamentados en la idea de que la escritura refleja el estado de ánimo y otros rasgos de la persona, se espera que el contenido emocional expresado en los mensajes que envían los progenitores varíe en función de estas dos variables de estudio.

En este sentido, aunque desconocemos estudios que analicen la relación entre el estado de ánimo y la adquisición de la discapacidad, algunos autores sí se han dirigido al análisis de las emociones y su asociación con algunas variables relativas a la discapacidad como la tipología de esta. Por ejemplo, Carroll (2008) examinó el lenguaje cognitivo y emocional utilizado en las narraciones de los padres de niños con síndrome de Down en comparación con otras discapacidades del desarrollo, reflejándose que los padres de niños con síndrome de Down hacían más referencias a las emociones positivas.

En los resultados del presente estudio se observa que el estado emocional que expresan los progenitores en sus mensajes es diferente según tengan hijos con discapacidad adquirida o sobrevenida, observándose que los mensajes intercambiados en los foros de padres y madres con hijos que adquirieron la discapacidad en el nacimiento reflejan connotaciones más positivas que las contribuciones enviadas por los progenitores a los foros con discapacidad sobrevenida. Por tanto, parece que el momento en el que se adquiere la discapacidad es una variable influyente en el estado de ánimo, manifestándose un estado emocional más positivo en los padres de hijos con discapacidad adquirida en el nacimiento. Esta idea queda fortalecida al examinar las dimensiones negativas, en las que se observa una mayor frecuencia de mensajes con sentimientos negativos, emociones de enfado, tristeza e ira, en los mensajes intercambiados en los foros dirigidos a padres y madres de hijos con discapacidad sobrevenida. Esto está en concordancia con autores como Uppal (2006) o Ford et al. (2001), que sugieren que el momento en el que se adquiere la discapacidad es una variable que afecta de forma diferente a la adaptación familiar a esta situación, siendo más compleja cuando sobreviene a lo largo de la vida.

Así, aunque es cierto que cuando nace un hijo con discapacidad el acontecimiento suele ser impactante y va a repercutir a lo largo de todo el ciclo vital, los progenitores desarrollan paralelamente al crecimiento del hijo unas estrategias de afrontamiento que les permiten superar las barreras con las que han de enfrentarse día a día, normalizando la difícil situación e incluso convirtiendo esta en una experiencia recompensante (Wehmeyer y Field, 2007). Sin embargo, cuando los padres se enfrentan a la discapacidad sobrevenida de un hijo, se encuentran con una situación diferente, ya que previamente habían pasado por un proceso de crianza normalizado y ahora se tienen que enfrentar a un doble proceso, uno de desadaptación al estilo de vida anteriormente llevado y que deben cambiar por la nueva situación y el otro, un proceso de afrontamiento-aprendizaje de otra forma de vida más traumática, con nuevas pautas 
de adaptación y, por tanto, más difícil de asimilar (Goodley y Tregaskis, 2006; Olsson y Hwang 2008; Suriá, 2013).

El segundo rasgo analizado que se ha asociado a un estado anímico es el tiempo verbal en que uno se expresa. Así, por ejemplo, Kramer, Guillory y Hancock (2014) sugieren que el tiempo verbal al que hacen referencia los individuos se asocia con su ajuste social y emocional, de forma que aquellos que hablan en presente y pasado perciben tener un futuro limitado y objetivos sociales más restringidos. En esta dirección parece apuntar el mayor número de referencias al presente en los mensajes enviados por los participantes de hijos con discapacidad sobrevenida. A su vez, este grupo presenta un menor porcentaje de mensajes en futuro que los participantes del foro dirigido a padres y madres de hijos con discapacidad adquirida en el nacimiento.

Con respecto al uso de pronombres personales, parece que el mayor empleo de pronombres en primera persona del singular y un menor uso de pronombres en segunda y tercera persona es característico de estados emocionales negativos (Pennebaker et al., 2003; Ramírez-Esparza et al., 2009; Tausczik y Pennebaker, 2010). Los resultados respaldan la hipótesis de que los progenitores de hijos con discapacidad sobrevenida expresan un estado de ánimo más negativo, puesto que se observa un mayor porcentaje de pronombres en primera persona entre los mensajes escritos por los progenitores de hijos con discapacidad sobrevenida. Asimismo, se observa menor proporción de pronombres en segunda y tercera persona en los mensajes enviados por los participantes de hijos con discapacidad sobrevenida.

Al examinar el contenido emocional en función de la otra variable del estudio, esto es, del género de los progenitores, se observa que las mujeres intercambian mensajes con connotaciones más positivas que los varones. En este sentido, la mayor parte de la literatura existente sobre las diferencias encontradas en la temática de los mensajes intercambiados en los grupos virtuales en función del género indica que varones y mujeres suelen expresarse de forma distinta en los mensajes que escriben, encontrándose que el género masculino escribe en mayor medida mensajes de tipo más negativo, mientras que las mujeres tienden a intercambiar contribuciones relativas a sentimientos positivos (Mehl y Pennebaker, 2003; Newman, Groom, Handelman y Pennebaker, 2008; Pennebaker et al., 2003; Tausczik y Pennebaker, 2010). Esta idea es perfectamente compatible con los estereotipos de género. En los resultados de este estudio esto queda reflejado, ya que se observa que no solo son las mujeres las que indican más emociones positivas, sino que los varones muestran sentimientos de enfado o ira.

Del mismo modo, los varones muestran mayor frecuencia en el uso de pronombres en primera persona del singular. Estos datos también están de acuerdo con los hallazgos de otros autores como Mulac, Bradac y Gibbons (2001), los cuales reflejan que los varones utilizan los pronombres en primera persona singular con más frecuencia que las mujeres.

Finalmente, los varones indican un mayor porcentaje de palabras expresadas en pasado. Como apuntan los resultados esto está en concordancia con la idea de un estado de ánimo más negativo en los padres que en las madres de hijos con discapacidad.

Por tanto, y a partir de estos resultados, es evidente que la vivencia de cuidar a un hijo con discapacidad es una experiencia de gran intensidad emocional, a la que 
multitud de padres y madres deben hacer frente de manera cotidiana, enfrentándose a obstáculos que, en muchas ocasiones, vulneran el estado de ánimo y de salud. De esta forma, una de las estrategias que puede ayudar a profundizar en la experiencia de estos padres y madres que conviven con la discapacidad es precisamente a través del análisis de sus expresiones emocionales.

Sin embargo, aunque pueden resultar interesantes los resultados de este estudio, se deben mencionar algunas limitaciones. La primera es que no se debe olvidar que la experiencia de la discapacidad es única para cada persona ya que está influida por una compleja combinación de factores (desde las diferencias personales de experiencias, temperamentos y contextos diferentes), por tanto, deberíamos profundizar en multitud de variables que quedan por tener en cuenta. Por otra parte, el LIWC es una herramienta que permite el rápido análisis de una gran cantidad de palabras, sin embargo, captura el sentido literal de las expresiones y tampoco tiene en cuenta la influencia del contexto en el discurso. A partir de estas consideraciones, en un futuro sería interesante acompañar los resultados con entrevistas y relatos de los progenitores que atienden día a día a un hijo con discapacidad para completar la información sobre la experiencia de cuidar a un hijo con discapacidad.

\section{Referencias bibliográficas}

Alpers, G. W., Winzelberg, A. J., Classen, C., Roberts, H., Dev, P., Koopman, C. y Taylor, C. B. (2005). Evaluation of computerized text analysis in an Internet breast cancer support group. Computers in Human Behavior, 21 (2), 361-376.

Amaya, R., Celene, A., Acle Tomasini, G., Ampudia Rueda, A. y García Méndez, M. (2014). Caracterización de los conocimientos de las madres sobre la discapacidad de sus hijos y su vínculo con la dinámica familiar. Acta Colombiana de Psicología, 17 (1), 91-103.

AzAR, M. y KuRdahi, L. (2006). The adaptation of mothers of children with intellectual disability in Lebanon. Journal of Transcultural Nursing, 17 (4), 375-380.

BeléNDEZ, M. y SuRiÁ, R. (2011). Los foros de Internet para trastornos de ansiedad y depresión: un análisis comparativo del uso del lenguaje. Ansiedad y Estrés, 17, 15-25.

Benítez, Y. G. y Soto, E. G. (2012). Las familias ante la discapacidad. Revista Electrónica de Psicología Iztacala, 15 (3), 1023.

Bonanno, G. A., Kennedy, P., Galatzer-Levy, L. R. y Lude, P. (2012). Trajectories of Resilience, Depression, and Anxiety Following Spinal Cord Injury. Rehabilitation Psychology, 57 (3), 236-247.

Cavicchio, F. y Poesio, M. ( 2012). The Rovereto Emotive Corpus: a new resource to investigate the pragmatics of emotions. Language Resources and Evaluation, 46, 117-130.

CoHEn, J. (1988). Statistical power analysis for the behavioral sciences. New Jersey: Lawrence Erlbaum.

Carrera-Fernández, M. J., Olmos, J. G. y Peró, M. (2013). Psicología y lenguaje en política: los candidatos a la Presidencia del Gobierno y su estilo lingüístico. Anuario de Psicología, 43 (1), 39-52.

CARroll, D. W. (2008). Books written by parents of children with developmental disabilities: A quantitative analysis. Journal on Developmental Disabilities, 14, 9-18.

Carroll, D. W. (2009). Cognitive and Emotional Language Use in Parents of Children with Developmental Disabilities. Journal on Developmental Disabilities, 15 (3), 88-95. 
Dabrowska, A. y Pisula, E. (2010). Parenting stress and coping styles in mothers and fathers of pre-school children with autism and Down syndrome. Journal of Intellectual Disability Research, 54, 266-280.

Dunst, C. J. y TrivetTe, C. M. (2009). Capacity building family systems intervention practices. Journal of Family Social Work, 12 (2), 119-143.

Einfeld, S. L., Stancliffe, R. J., Gray, K. M., Sofronoff, K., Rice, L., Emerson, E. y Yasamy, M. T. (2012). Interventions provided by parents for children with intellectual disabilities in low and middle income countries. Journal of Applied Research in Intellectual Disabilities, $25,135-142$.

Ford, H. L., Gerryoe, E. y Tennant, A. (2001). Health status andquality of life of people with multiple sclerosis. Disability and Rehabilitation, 23 (12), 516-521.

Goodley, D. y Tregaskis, C. (2006). Storying disability and impairment: Retrospective accounts of disabled family life. Qualitative Health Research, 16 (5), 630-646.

Graesser, A. C., Dowell, N. y Moldovan, C. (2011). A computer's understanding of literature. Scientific Studies of Literature, 1, 24-33.

Jordán de Urríes, F. B. (1997). Desarrollo del Cuestionario Parental de Diagnóstico Subjetivo CPDS. Relaciones entre el Diagnóstico Subjetivo de los padres y diferentes variables referentes a sus hijos con Autismo o Parálisis Cerebral. Siglo Cero, 28 (4), 35-46.

Kramer, A. D., Gulllory, J. E. y Hancock, J. T. (2014). Experimental evidence of massivescale emotional contagion through social networks. Proceedings of the National Academy of Sciences, 111 (24), 8788-8790.

Krethong, P., Jirapaet, V., Jitpanya, C. y Sloan, R. (2008). A Causal Model of Health Related Quality of Life in Thai Patients with Heart-Failure. Journal of Nursing Scholarship, 40 (3), 254-260.

Lequerica, A. H., Forchheimer, M., Albright, K. J., Tate, D. G., Duggan, C. H. y Rahman, R. O. (2010). Stress Appraisal in Women with Spinal Cord Injury: Supplementary Findings Through Mixed Methods. International Journal of Stress Management, 17 (3), 259-275.

Mehl, M. R. y Pennebaker, J. W. (2003). The sounds of social life: A psychometric analysis of students' daily social environments and natural conversations. Journal of Personality and Social Psychology, 84, 857-870.

Mulac, A., Bradac, J. y Gibbons, P. (2001). Empirical support for the gender as culture hypothesis. Human Communication Research, 27 (1), 121-152.

Newman, M. L., Groom, C. J., Handelman, L. D. y Pennebaker, J. W. (2008). Gender differences in language use: An analysis of 14,000 text samples. Discourse Processes.

Olsson, M. B. y Hwang, C. P. (2008). Socioeconomic and psychological variables as risk and protective factors for parental well-being in families of children with intellectual disabilities. Journal of Intellectual Disability Research, 52, 1102-1113.

Pennebaker, J. W., Francis, M. E. y Booth, R. J. (2001). Linguistic Inquiry and Word Count (LIWC): LIWC. Mahwah, New York: Erlbaum Publishers.

Pennebaker, J. W., Mehl, M. R. y Niederhoffer, K. (2003). Psychological aspects of natural language use: Our words, our selves. Annual Review of Psychology, 54, 547-577.

Price, S., Price, C. H. y McKenry, P. (2010). Families y Change. Coping with stressful events and transitions. Sage Publications Inc: USA.

Ramírez-Esparza, N., Chung, C. K., Sierra-Otero, G. y Pennebaker, J. W. (2009). El lenguaje de la depresión: Categorías lingüísticas y temas usados en foros de discusión en internet en inglés y en español. Revista de la Asociación de Psicoterapia de la República Argentina, 2, 1-28. 
Ramírez-Esparza, N., García, F., Pennebaker, J. W. y Suriá, R. (2007). La psicología del uso de las palabras. Un programa de ordenador que analiza textos en inglés y en español. Revista Mexicana de Psicología, 24, 85-99.

Robinson, R. L., Navea, R. e Ickes, W. (2013). Predicting final course performance from students' written self-introductions: A LIWC analysis. Journal of Language and Social Psychology, 32 (4), 469-479.

Rude, S. S., Gortner, E. M. y Pennebaker, J. W. (2004). Language use of depressed and depression vulnerable college students. Cognition E Emotion, 18, 1121-1133.

Samios, C., Pakenham, K. I. y Sofronoff, K. (2009). The nature of benefit finding in parents of a child with Asperger syndrome. Research in Autism Spectrum Disorders, 3 (2), 358-374.

Schalock, R. L., Bohman, G. S. y Verdugo, M. Á. (2008). The conceptualization and measurement of quality of life: Implications for program planning and evaluation in the field of intellectual disabilities. Evaluation and Program Planning, 31, 181-190.

Schwartz, H. A., Eichstaedt, J. C., Kern, M. L., Dziurzynski, L., Ramones, S. M., Agrawal, M. ... y Ungar, L. H. (2013). Personality, gender, and age in the language of social media: The open-vocabulary approach. PloS one, 8 (9), e73791.

Singer, G., Ethridge, B. y Sandra, A. (2007). Primary and secondary effects of parenting and stress management interventions for parents of children with developmental disabilities: a metaanalysis. Mental Retardation and Developmental Disabilities Research Reviews, 13, 357-369.

Slatcher, R. B., Vazire, S. y Pennebaker, J. W. (2008). “Am 'I' more important than 'we'? Couples' word use in instant messages”. Personal Relationships, 15, 407-424.

Smith, L., Hong, A., Seltzer, M., Greenberg, J., Almeida, D. y Bishop, S. (2010). Daily experiences among mothers of adolescents and adults with ASD. Journal of Autism and Developmental Disorders, 40 (2), 167-178.

Stephen, R. y Keating, D. M. (2011). The Social Dimension of Blogging about Health: Health Blogging, Social Support, and Well-Being. Communication Monographs, 78 (4), 511-534.

Stirman, S. W. y Pennebaker, J. W. (2001). Word use in the poetry of suicidal and nonsuicidal poets. Psychosomatic Medicine, 63 (4), 517-522.

SuRIÁ, R. (2011). Discapacidad adquirida y discapacidad sobrevenida: análisis comparativo de la sobrecarga que generan ambas formas de discapacidad en las madres de hijos afectados. Siglo Cero, 42, 67-84.

SURIÁ, R. (2013). Análisis comparativo de la fortaleza en padres de hijos con discapacidad en función de la tipología y la etapa en la que se adquiere la discapacidad. Anuario de Psicología, 43, 23-37.

SURIÁ, R. (2015). Profiles of resilience and quality of life in people with acquired disability due to traffic accidents. Gaceta Sanitaria, 29, 55-59.

SuRIÁ, R. y Beléndez, M. (2009). El efecto terapéutico de los grupos virtuales para pacientes con enfermedades crónicas: evolución del contenido emocional de los mensajes de sus participantes. Boletín de Psicología, 96, 35-46.

Tausczik, Y. R. y Pennebaker, J. W. (2010). The psychological meaning of words: LIWC and computerized text analysis methods. Journal of language and social psychology, 29 (1), 24-54.

Uppal, S. (2006). Impact of the timing, type and severity of disability on the subjective wellbeing of individuals with disabilities. Social Science y Medicine, 63, 525-539.

UrzúA, A., Godoy, J. y Ocayo, K. (2011). Competencias parentales percibidas y calidad de vida. Revista Chilena de Pediatría, 82 (4), 300-310. 
VAldés-SÁnchez, N. (2010). Análisis de los estilos lingüísticos de paciente y terapeuta durante la conversación terapéutica en episodios de cambio, utilizando el Buscador Lingüístico y Contador de Palabras (LIWC). Subjetividad y Procesos Cognitivos, 14 (2), 314-332.

Van Uden-Kraan, C. F., Drossaert, C. H., Taal, E., Smit, W. M., Seydel, E. R. y Van de LAAr Ma, F. J. (2008). Coping with somatic illnesses in online support groups. Do the feared disadvantages actually occur? Computers in Human Behavior, 24, 309-324.

Wehmeyer, M. L. y Field, S. (2007). Self-determination: Instructional and assessment strategies. Thousand Oaks, CA: Corwin Press.

Wright, K. B., Rains, S. A. y BANAs, J. A. (2010). Weak tie support network preference and computer-mediated support groups. Journal of Computer-Mediated Communication, 15, 606-624. 